\title{
Taura syndrome virus (TSV) lesion development and the disease cycle in the Pacific white shrimp Penaeus vannamei
}

\author{
Kenneth W. Hasson*, Donald V. Lightner, Leone L. Mohney, Rita M. Redman, \\ Bonnie T. Poulos, Brenda M. White
}

Department of Veterinary Science and Microbiology, University of Arizona, Tucson, Arizona 85721, USA

\begin{abstract}
The Taura syndrome virus (TSV) disease cycle was redefined through histological and gene probe analysis of experimentally infected specific pathogen-free (SPF) Penaeus vannamei sampled at timed intervals. The cycle consists of 3 overlapping, but clinically and histologically distinct, phases: a $\sim 7 \mathrm{~d}$ peracute to acute phase, a $\sim 5 \mathrm{~d}$ transition phase (previously termed the chronic or recovery phase), and a definitive chronic phase. The acute phase is characterized by the rapid development of severe, multifocal to diffuse cuticular epithelial necrosis and high mortalities. Using in situ hybridization analysis, infected pre-lytic cuticular epithelial cells display very strong TSV-positive probe signals, and a total of 3 stages of acute phase necrosis are described. Surviving $P$. vannamei then enter the transition phase, which is distinguished histologically by multifocal melanized lesions within regions of the cuticular epithelium (resolving acute phase lesions), focal active acute phase lesions, and the onset of lymphoid organ (LO) spheroid development. Gene probe analysis of transitionally infected shrimp reveals probe-positive foci of active acute phase lesions, a diffuse probe signal within the walls of morphologically normal LO tubules and/or focal probe signals within developing LO spheroids. Shrimp surviving this stage enter the chronic phase infection after ecdysis. The defining characteristics of the chronic phase include the cessation of mortalities, the resumption of normal behavioral patterns, the complete absence of visible melanized lesions and acute phase histological lesions of the cuticular epithelium, and marked LO hypertrophy directly resulting from the rapid development of numerous LO spheroids, some of which are TSV positive by in situ hybridization analysis.
\end{abstract}

KEY WORDS: Taura syndrome virus (TSV) - Picornaviridae $\cdot$ Penaeid shrimp In situ hybridization

\section{INTRODUCTION}

During 1994, the penaeid shrimp disease Taura syndrome (TS) was demonstrated to be caused by a previously undescribed virus (Hasson et al. 1995), which was named Taura syndrome virus or TSV (Brock et al. 1995). Characterization work by Bonami et al. (1997) established that TSV was a possible member of the Picornaviridae. Since the initial recognition of TS disease in Ecuador during 1992 (Jimenez 1992), TSV has spread into Penaeus vannamei (suggested genus name change from Penaeus to Litopenaeus, Perez Farfante \&

•E-mail: hasson@primenet.com
Kensley 1997) farming regions of 13 different countries: Ecuador, Brazil, Colombia, Peru, Panama, Belize, Costa Rica, Nicaragua, El Salvador, Honduras, Guatemala, Mexico, and the United States (Lightner 1996a,b, Brock et al. 1997. Lightner et al. 1997. Hasson 1998, Hasson et al. 1999). Cumulative economic loss due to TSV epizootics throughout the Americas has been estimated to be between $\$ 1.2$ and $\$ 2$ billion for the years 1992 to 1996 (Lightner 1995, 1996b, Hasson 1998). Of the 13 countries that have been adversely impacted by the disease, Belize is the only country, thus far, to have reported successful eradication of the virus from its shrimp growing regions (Dixon \& Dorado 1997).

Based on early histological investigations of Penaeus vannamei with naturally occurring TSV infections, the 
TSV disease cycle was originally described as having 2 distinct phases: a peracute to acute phase and a separate chronic phase (Lightner et al. 1994, 1995a, Brock et al. 1995, Hasson et al. 1995). During the acute phase of a TSV infection, the cuticular epithelium and subcutis of the gills, foregut, hindgut, appendages and general body cuticle are targeted by the virus (Jimenez 1992, Lightner et al. 1994, 1995, Brock et al. 1995, Hasson et al. 1995). The pathodiagnostic lesion type that results is characterized by necrotic cuticular epithelial cells, which display cytoplasmic eosinophilia, nuclear pyknosis and karyorrhexis, and the formation of variably sized and variably staining cytoplasmic inclusion bodies (Lightner et al. 1994, 1995). In cases of severe acute phase TSV infections, this lesion type has been observed to extend into the subcuticular connective tissue and/or striated muscle (Lightner et al. 1994, 1995, Brock et al. 1995, 1997).

The clinical signs of an acute phase TSV infection in Penaeus vannamei include anorexia, lethargy, atactic swimming behavior, opaque musculature, chromatophore expansion (pale red to lavender body coloration in naturally occurring infections and pale grey coloration in experimentally induced infections) and soft cuticles. In general, death occurs in 75 to $95 \%$ of an infected population within a week of disease onset (Lightner et al. 1994, Brock et al. 1995, Hasson et al. 1995, Lightner 1996a). Surviving $P$. vannamei then develop variably sized and shaped melanized cuticular lesions or brown spots, which are grossly visible over portions of the tail, cephalothorax and appendages (Lightner et al. 1994, Brock et al. 1995, Hasson et al. 1995, Lightner 1996a). These non-specific Iesions are similar in appearance to those caused by chitinolytic Vibrio sp. (shell disease) and were previously considered to be the hallmark of a chronic TSV infection (Lightner et al. 1994, 1995, Brock et al. 1995, 1997, Hasson et al. 1995, Lightner 1996a, Brock 1997). Histologically, these lesions typically consist of abundant hemocytic infiltrates (inflammation) and in some, but not all, cases secondary Vibrio sp. colonization of the exocuticle. Subsequent histological analyses of experimentally infected $P$. vannamei juveniles led Hasson et al. (1995) to hypothesize that these 'chronic phase' lesions were representative of resolving acute phase TSV lesions and that the term 'recovery phase' may be better suited for describing this stage of the TSV infection cycle. Hasson et al. (1995) speculated that the formation of spheroids within the lymphoid organ was directly attributable to TSV infection, contrary to the belief that these lesions were representative of a concurrent secondary infection by lymphoid organ vacuolization virus or LOVV (Jimenez 1992). During the time that these observations were made, TSV-specific genomic probes were developed by Mari et al. (1998) and further analysis of TSV lesion pathogenesis was postponed until this new technology could be employed. Utilizing the technique of in situ hybridization analysis for the detection of TSV, the present study was conducted to determine (1) which tissues or regions of the shrimp host are initially infected, (2) if specific tissues or regions are preferentially infected, (3) if lesion development within known target tissues occurs at random or follows a set pattern, (4) the approximate time frame of both the acute and chronic phase infections and (5) to document virus-induced morphological changes within the cuticular epithelium and lymphoid organ.

\section{MATERIALS AND METHODS}

Experimental shrimp. Specific pathogen-free (SPF) Penaeus vannamei from the Oceanic Institute's SPF breeding center (Hawaii) were reared and maintained following the criteria of both Wyban et al. (1992) and Pruder et al. (1995). Approximately 1 mo prior to initiating this study, the shrimp were air shipped from Hawail to the University of Arizona, where they were maintained in two $1500 \mathrm{l}$ fiberglass tanks with recirculating, biofiltered artificial seawater (Forty Fathoms Biocrystals Marinemix, Marine Enterprises International Inc., Baltimore, MD).

A total of 600 SPF Penaeus vannamei early juveniles (0.35 g avg. wt) were equally distributed among six 901 glass aquaria. Each aquarium was equipped with a recirculating 1 l oyster shell biofilter to remove dissolved ammonia and nitrites. The aquaria were filled ( $\sim 80$ to 85 l) with artificially prepared seawater (20 ppt salinity) and water temperatures were maintained at $\sim 28^{\circ} \mathrm{C}$ by the installation of a submersible heater in each aquarium. Each aquarium was covered with a plastic sheet to prevent the escape of the test shrimp and to contain TSV-contaminated aerosols.

Virus exposure and shrimp sample collection. The 5 treatment groups were exposed to TSV by feeding minced Penaeus vannamei carcasses collected during a naturally occurring TSV epizootic in Texas in 1995 (courtesy of Mr. Fritz Jaenike, Harlingen Farms). Infected tissue was fed at a rate of $34 \%$ of the biomass per aquarium (12 g) divided between 2 daily feedings (08:00 and 14:00 h) during the first $3 \mathrm{~d}$ of the $10 \mathrm{~d}$ study. Following this same feeding regime, the single negative control group was fed TSV-free SPF $P$. vannamei minced tissue. Beginning on Day 4, all shrimp were fed a pelleted ration (Rangen No.4), ad libitum, twice daily.

Four of the 5 treatment groups were sampled at timed intervals by collecting 2 shrimp at random from each aquarium every $3 \mathrm{~h}$ following the first per os exposure at 08:00 h on Day 1. Sampling was then in- 
creased to 3 shrimp aquarium ${ }^{-1}$ at $3 \mathrm{~h}$ intervals on Day 2 and continued for the first 6 of the 8 sampling periods of Day 3. Starting with the last two $3 \mathrm{~h}$ sampling intervals of Day 3 and through Day 4 , the number of shrimp collected per treatment aquarium was reduced to 2. Beginning on Day 5 until termination on Day 10, 2 shrimp were collected from each of the 4 treatment aquaria per day. This sampling schedule was conducted to obtain the maximum number of shrimp with early peracute stage infections without depleting the infected stocks prior to the end of the $10 \mathrm{~d}$ period. The fifth TSV treatment group served as a positive control and was only sampled when moribund shrimp were observed in order to determine the daily and cumulative percent mortality resulting from the virus-caused epizootic. Five samples of the single negative control group were preserved, prior to the onset of the experiment and, again, upon termination. Time course sampling of this group was not performed in order to determine the cumulative percent mortality of the population in the absence of TSV. The shrimp samples were injected with R-F (RNA-friendly) fixative following the methods of Hasson et al. (1997). The shrimp collected during each sampling interval from the 4 treatment aquaria were pooled. After $48 \mathrm{~h}$ in R-F fixative, the samples were transferred to $70 \%$ ethanol for $24 \mathrm{~h}$ and then processed for histological analysis following the methods of Bell \& Lightner (1988).

Histopathology and in situ hybridization analyses. A paraffin block of each shrimp sample was prepared to include a portion of the gills and midsagittal sections of both the cephalothorax and the sixth tail segment (hindgut location). Efforts were made to obtain midsagittal sections of the cephalothorax, which displayed both the foregut and the lymphoid organ. Following embedding, 4 to $5 \mu \mathrm{m}$ consecutive sections of each sample were prepared. One section per sample was placed on a poly-L-lysine coated slide (Sigma, St. Louis, $\mathrm{MO}$ ) for routine histological analysis and the corresponding consecutive section collected on a silane coated slide (Silane-Prep, Sigma) for in situ hybridization analysis. Sections for routine histology were stained with Mayer-Bennett hematoxylin-eosin phloxine following standard methods (Bell \& Lightner 1988, Lightner 1996a). In situ hybridization analyses were performed using a 1:1 mixture of 2 TSV-specific, digoxigenin (DIG)-labeled cDNA genomic probes following the methods of Hasson et al. (1997) and Mari et al. (1998). With the exception of wearing latex gloves, no RNase-free precautions were used in the preparation of the reagents or in conducting the assays.

In situ hybridization assays were conducted on 270 of the 361 shrimp samples collected. A maximum of 24 samples were assayed during a given in situ hybridization run, and the same lot of DIG-labeled TSV probe was used for each assay. Both TSV-negative and TSV-positive control Penaeus vannamei histological sections were included in each assay. All sections were counter-stained with Bismarck brown, coverslipped, and examined by brightfield microscopy following the methods of Lightner (1996a). The number of TSVinfected tissues or organs present in each specimen was determined based on the observation of a gene probe positive signal (blue-black precipitate). The presence of TSV within a given organ or tissue was qualitatively graded from 0 to 4 according to the modified grading system of Bell \& Lightner (1987) and Hasson et al. (1995). The absence of a TSV gene probe signal was graded as 0 ; mild, focal signals were graded as 1 ; moderate, locally extensive to multifocal probe signals were assigned a grade of 2 to 3 , and numerous multifocal to diffuse signals were assigned a grade of 4 . Overall infection severity per shrimp was based on the single tissue or region that displayed the most extensive gene probe signal.

\section{RESULTS}

Cumulative percent mortalities in the positive and negative Penaeus vannamei control groups were 85 and $23 \%$, respectively (Table 1 ). The mortalities which occurred in the negative control group were attributed to cannibalism of recently molted shrimp, as histological and in situ hybridization analyses of 5 shrimp, collected on Days 0 and 10, revealed no detectable viral or bacterial infections, nor signs of noninfectious diseases. Mortalities among the positive control group began within $24 \mathrm{~h}$ post-exposure, peaked on Day 4 and then rapidly declined, ending by Day 8 (Fig. 1). Moribund test shrimp displayed the same clinical signs of an experimentally induced TSV infection as described earlier and previously reported by Hasson et al. (1995). Typically, moribund treatment shrimp would die during the final stage of molting or immediately post-molt, which accounted for their soft-shell texture. Beginning on Day 4 post-exposure and until the experiment was terminated on Day 10, a few of the remaining treatment shrimp presented grossly visible multifocal, variably sized and shaped, brownish-black (melanized) lesions on both the cephalothoraxic and tail regions. Histological and gene probe analysis of 5 moribund positive control shrimp, collected during the peak dieoff on Day 4 (Table 1), showed that each contained severe $(G 3-4)$ pathodiagnostic TSV lesions of the cuticular epithelium and indicated that the tissue fed to all of the treatment groups contained infectious TSV.

Of the 270 treatment shrimp analyzed for the presence of TSV by in situ hybridization, 92 were found to be infected (Table 2). Seventy (76\%) of the 92 probe- 
Table 1. Penaeus vannamei. Daily and cumulative percentage mortalities of juvenile positive and negative control groups. Test shrimp were exposed per os to either TSV-infected (positive ctrl) or specific pathogen-free minced $P$. vannamei tissue (negative ctrl) during Days 1 through 3 of the study

\begin{tabular}{|c|c|c|c|c|c|c|c|c|c|c|c|c|c|c|c|}
\hline \multirow[t]{2}{*}{ Treatment } & \multicolumn{14}{|c|}{ Daily mortality (d) } & \multirow{2}{*}{$\begin{array}{l}\text { Cumulative } \\
\% \text { mortality }\end{array}$} \\
\hline & 0.5 & 1 & 1.5 & 2 & 2.5 & 3 & 3.5 & 4 & 5 & 6 & 7 & 8 & 9 & 10 & \\
\hline Negative ctrl & 1 & 1 & 0 & 0 & 0 & 0 & 0 & 1 & 3 & 1 & 0 & 1 & 0 & 0 & $23 / 100,23 \%$ \\
\hline Positive ctrl & 1 & 1 & 1 & 2 & 5 & 6 & 11 & 26 & 21 & 5 & 1 & 0 & 0 & 0 & $85 / 100,85 \%$ \\
\hline
\end{tabular}

positive samples demonstrated TSV within the cuticular epithelial cells of 1 or more of the 5 regions of the shrimp that contain this cell type and were classified as being acutely infected (Table 2). The 5 regions that were TSV infected included the appendages (maxillipeds, antennae, antennules, antennal scales, eyestalks, periopods and pleopods) general body cuticle, foregut (mouth, esophagus, anterior stomach, posterior stomach), gills (lamellae and mastigobranchiae) and hindgut (Fig. 2). The first detectable acute phase TSV infection was observed within the cuticular epithelial cells of the foregut and gills of 1 of the 8 treatment shrimp that were sampled $24 \mathrm{~h}$ following the first per os exposure (Table 2). An average of 3 of the 5 targeted regions displayed low grade to severe (G1-4) TSV infections during the first $2 \mathrm{~d}$ post-exposure. From Days 3 through 5, both the number of TSV-infected regions and acute phase lesion severity increased, affecting an average of 4 of the 5 targeted regions (Table 2).

The initial site of TSV infection within the samples was not determined, nor was a detectable preference shown by the virus for one region of cuticular epithelial cells over another. However, the cuticular epithelium of the foregut was found to have the highest infection prevalence, followed closely by that of the general body cuticle (Table 2 ). In contrast, the cuticular epithelium of the hindgut was found to have the lowest infection prevalence and TSV lesions were only apparent in this region when the shrimp displayed severe (G3-4) acute phase infections within 3 or more of the remaining 4 targeted regions.

Comparison of parallel H\&E histological and geneprobed sections showed that acutely infected cuticular epithelial cells display 3 stages of necrosis that can be observed by light microscopy (Fig. 3). Initially, the

Table 2. Penaeus vannamei. In situ hybridization results of time course sampled shrimp following per os exposure to TSV-infected tissue. Between 6 and 36 shrimp were analyzed per collection period and TSV-infected tissues were determined by the formation of a blue-black precipitate following gene probe analysis. Six regions of the test shrimp were infected by TSV and include the cuticular epithelium of the appendages (AP), body cuticle (BC), foregut (FG), gill lamellae (GL), hindgut (HG) and the lymphoid organ tubule cells (LO). Based on the regions infected per sample, the shrimp were assigned to 1 of 3 TSV infection categories: acute phase, TSV-positive probe signal(s) within the cuticular epithelium; transition phase, TSV-positive signal(s) within the cuticular epithelium and lymphoid organ; chronic phase, TSV-positive signal(s) within the lymphoid organ only

\begin{tabular}{|c|c|c|c|c|c|c|c|c|c|c|c|}
\hline \multirow{2}{*}{$\begin{array}{l}\text { Time of } \\
\text { sampling } \\
\text { (d) }\end{array}$} & \multirow{2}{*}{$\begin{array}{c}\text { No. of shrimp } \\
\text { analyzed by } \\
\text { in situ hybrization }\end{array}$} & \multirow[t]{2}{*}{$\begin{array}{l}\text { No. TSV } \\
\text { positive }\end{array}$} & \multicolumn{6}{|c|}{$\begin{array}{l}\text { Cumulative no. of tissues } \\
\text { infected per sample }\end{array}$} & \multicolumn{3}{|c|}{$\begin{array}{l}\text { Percent of TSV+ shrimp } \\
\text { assigned to each infection phase }\end{array}$} \\
\hline & & & $\mathrm{AP}$ & $\mathrm{BC}$ & $F G$ & GL & $\mathrm{HG}$ & LO & Acute & Transition & Chronic \\
\hline 0.5 & 24 & 0 & 0 & 0 & 0 & 0 & 0 & 0 & $0 \%$ & $0 \%$ & $0 \%$ \\
\hline 1 & 24 & 1 & 0 & 0 & 1 & 1 & 0 & 0 & $1 / 24,4 \%$ & $0 \%$ & $0 \%$ \\
\hline 1.5 & 29 & 2 & 2 & 2 & 2 & 1 & 0 & 0 & $2 / 29,7 \%$ & $0 \%$ & $0 \%$ \\
\hline 2 & 23 & 4 & 3 & 3 & 3 & 2 & 0 & 0 & $4 / 23,17 \%$ & $0 \%$ & $0 \%$ \\
\hline 2.5 & 28 & 4 & 1 & 2 & 2 & 3 & 0 & 0 & $4 / 28,14 \%$ & $0 \%$ & $0 \%$ \\
\hline 3 & 36 & 7 & 3 & 3 & 6 & 4 & 1 & 0 & $7 / 36,19 \%$ & $0 \%$ & $0 \%$ \\
\hline 3.5 & 32 & 22 & 13 & 10 & 21 & 8 & 2 & 0 & $22 / 32,69 \%$ & $0 \%$ & $0 \%$ \\
\hline 4 & 32 & 23 & 12 & 19 & 19 & 15 & 6 & 1 & $22 / 32,69 \%$ & $1 / 32,3 \%$ & $0 \%$ \\
\hline 5 & 8 & 4 & 1 & 2 & 2 & 2 & 0 & 2 & $2 / 8,25 \%$ & $2 / 8,25 \%$ & $0 \%$ \\
\hline 6 & 8 & 7 & 2 & 4 & 1 & 1 & 1 & 4 & $3 / 8,38 \%$ & $2 / 8,25 \%$ & $2 / 8,25 \%$ \\
\hline 7 & 8 & 4 & 2 & 4 & 4 & 1 & 1 & 1 & $3 / 8,38 \%$ & $1 / 8,13 \%$ & $0 \%$ \\
\hline 8 & 6 & 6 & 0 & 1 & 0 & 0 & 0 & 5 & $0 \%$ & $1 / 6,17 \%$ & $5 / 6,83 \%$ \\
\hline 9 & 6 & 3 & 0 & 0 & 0 & 0 & 0 & 3 & $0 \%$ & $0 \%$ & $3 / 6,50 \%$ \\
\hline 10 & 6 & 5 & 0 & 0 & 0 & 0 & 0 & 5 & $0 \%$ & $0 \%$ & $5 / 6,83 \%$ \\
\hline Total & 270 & 92 & 39 & 50 & 61 & 38 & 11 & 21 & 70 & 7 & 15 \\
\hline
\end{tabular}


Fig. 1. Penaeus vannamei. Daily mortality of SPF juveniles following per os exposure to either TSV-infected (positive control group) or pathogen-free (negative control group) minced $P$. vannamei carcasses

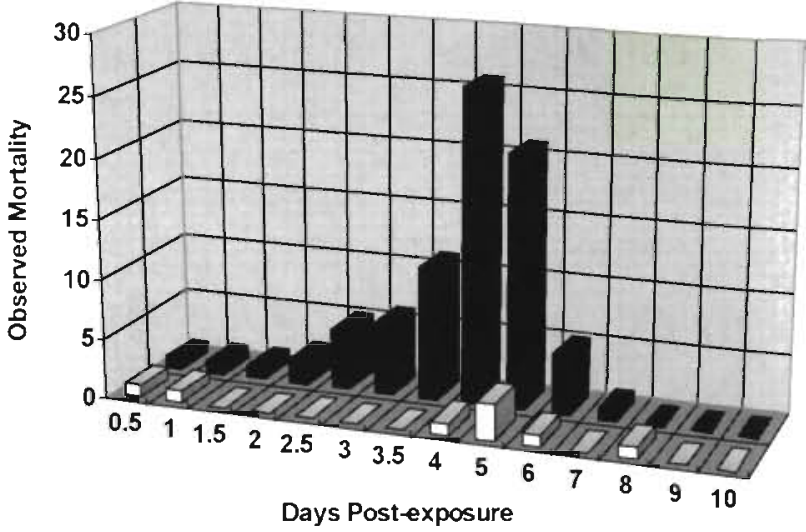

$\square$ Negative Control a Positive Control
Fig. 2. Penaeus vannamei. Photomicrographs of gene-probed histological sections illustrating the 5 cuticular epitheLial regions in SPF shrimp that are targeted by TSV during the acute phase of the infection cycle. Test shrimp were initially sampled at $3 \mathrm{~h}$ intervals following per os exposure to minced TSVinfected shrimp tissue during the first $3 \mathrm{~d}$ of this $10 \mathrm{~d}$ study. The presence of TSV RNA within cuticular epithelial cells is indicated by foci containing a black precipitate. (A) Distal end of a periopod (appendage), (B) dorsal cephalothorax (body cuticle), (C) and (D) esophagus and posterior stomach (anterior and posterior foregut), (E) gill lamellae, and $(F)$ hindgut. Sections were counter-stained with Bismarck brown Scale bars $=50 \mu \mathrm{m}$

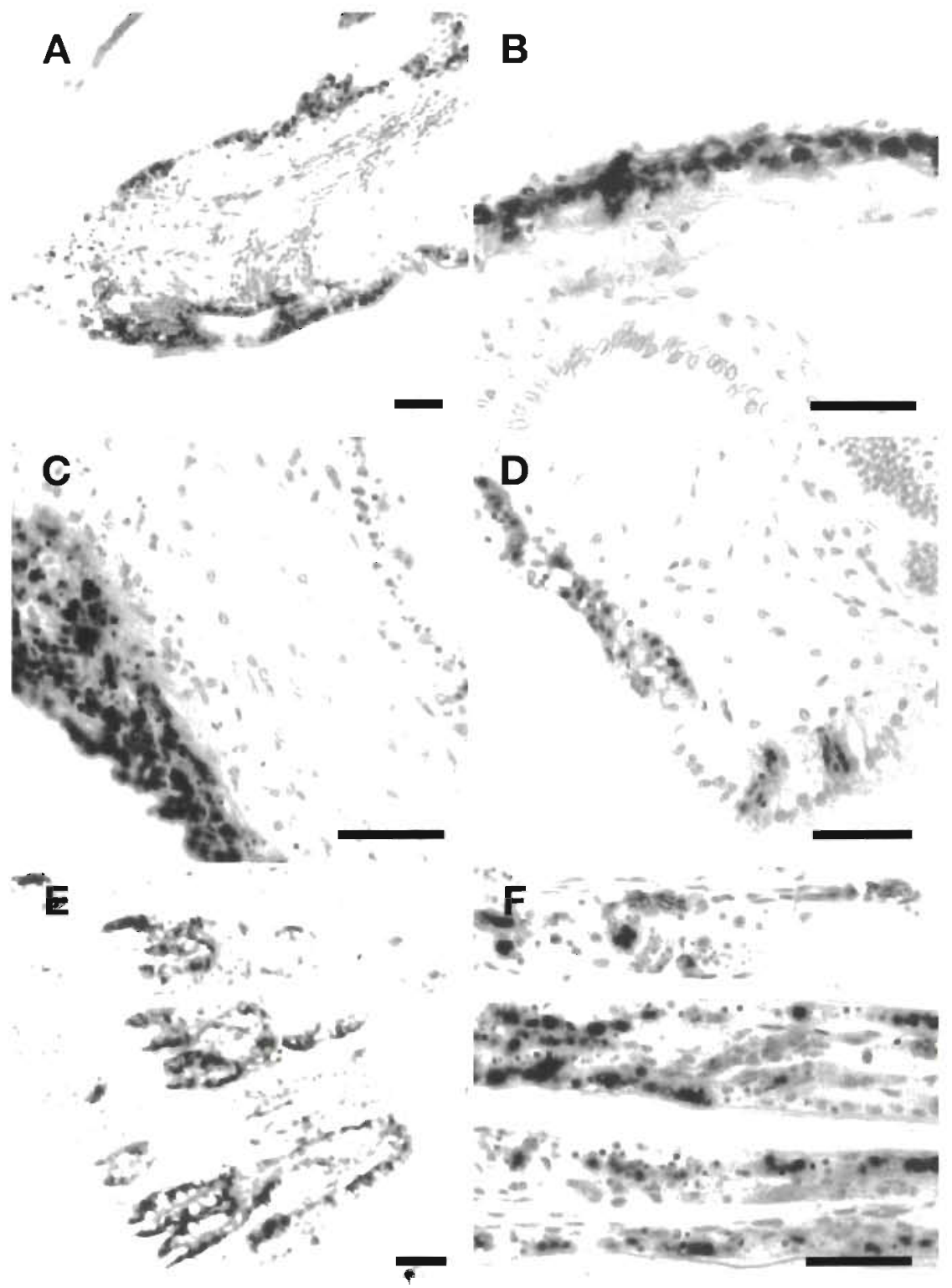




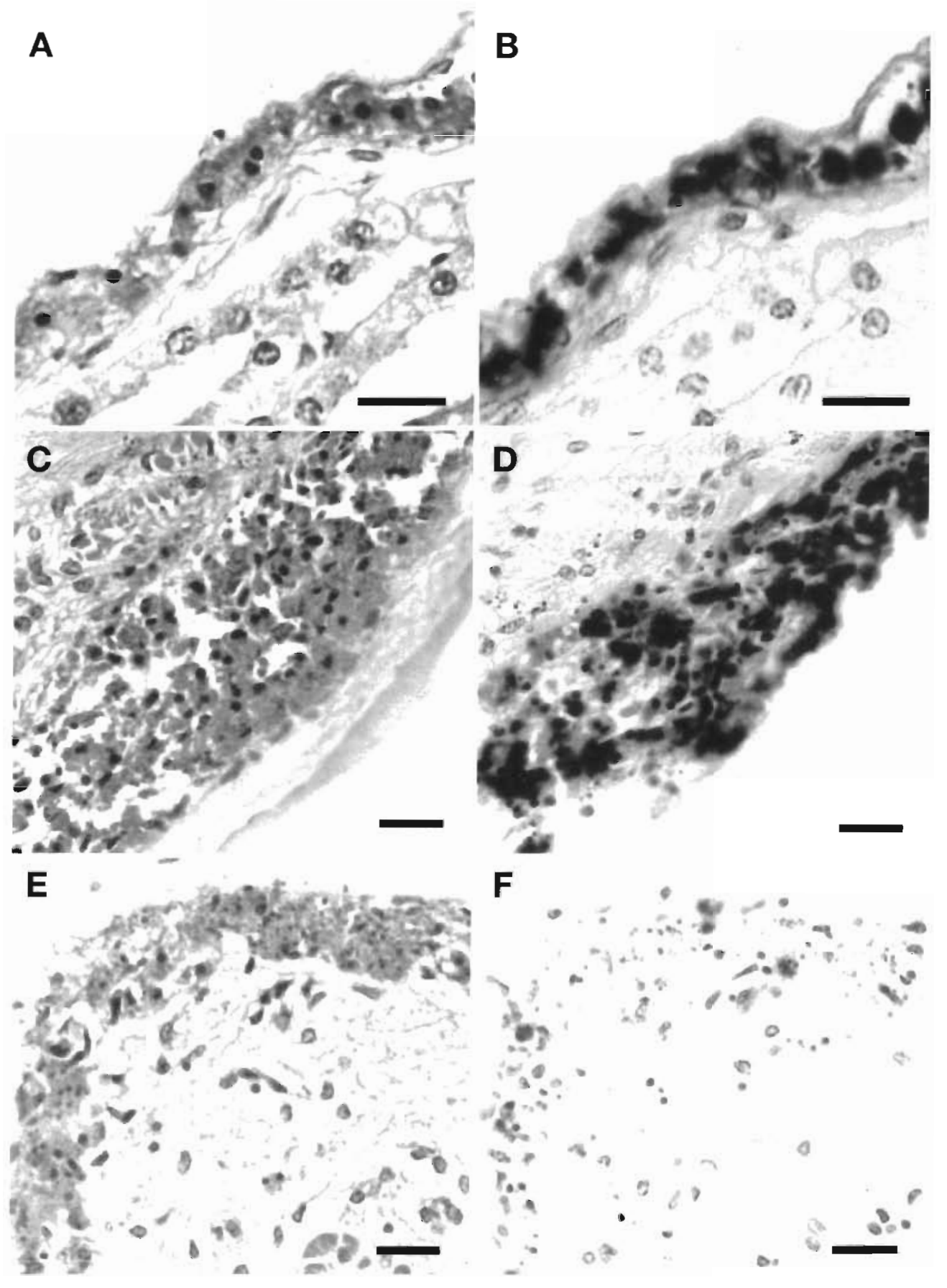

Fig. 3. Penaeus vannamej. Photomicrographs of parallel hematoxylin and eosin (H\&E)-stained (left column) and gene-probed (right column) histological sections illustrating the 3 stages of TSV-induced necrosis of the cuticular epithelium in acutely infected shrimp. The presence of TSV RNA within gene-probed sections is indicated by foci containing a black precipitate. (A, B) Stage 1 necrosis within cuticular epithelial cells located ventral to the nerve cord within the cephalothorax (body cuticle). Histologically, the infected cells display pyknotic nuclei and cytoplasmic eosinophilia (not apparent in this black and white photo). An intense gene probe signal is observed within the cytoplasm of these intact cells. (C, D) Stage 2 necrosis within cuticular epithelial cells of the esophagus. The infected cells have detached from the surrounding cell matrix, are spherical in appearance, remain intact, and continue to display nuclear pyknosis and cytoplasmic eosinophilia (not apparent in this black and white photo). TSV gene probe signals remain strong, as indicated by the black precipitate. (E, F) Stage 3 necrosis within cuticular epithelial cells of the esophagus. Cell lysis has occurred and pyknotic nuclei have fragmented (karyorrhectic nuclei). This is considered the pathodiagnostic lesion type of an acute phase TSV infection, which appears 'peppered' or 'buckshot laden' by routine histology. By in situ hybridization, the TSV probe signal of this lesion is weak or undetectable, as the virus has dispersed. Histological sections were stained with Mayer-Bennett H\&E. Gene-probed sections were counter-stained with Bismarck brown. Scale bars $=20 \mu m$ 
infected cells display increased eosinophilia of the cytoplasm and basophilic pyknotic nuclei (condensed chromatin, Fig. 3A). In the second stage, cytoplasmic eosinophilia is pronounced, the infected cell detaches from the surrounding cell matrix, circularizes, and appears hypertrophied (Fig. 3C). In the third and final stage of cell death, the nuclear and cytoplasmic membranes are presumed to rupture and the pyknotic nuclei fragment into smaller spheres (karyorrhexis, Fig. 3E). It is during this final stage that TSV-infected tissues display the characteristic 'peppered' or 'buckshot laden' appearance that is considered pathodiagnostic for the acute phase of the disease. The 'peppered' appearance results, in part, from the presence of both highly basophilic pyknotic and karyorrhectic nuclei and the formation of variably sized, variably staining spherical inclusion bodies. However, it is unclear by light microscopy whether these inclusion bodies are of viral origin or remnants of the host cell. Analysis of the 3 stages of necrosis by in situ hybridization showed that TSV is most readily detected in cuticular epithelial cells during Stages 1 and 2 (Fig. 3B,D). Once cell lysis has occurred, it is believed that the liberated viral particles are rapidly disseminated by the hemolymph, resulting in either a very low grade or undetectable TSV gene probe signal within the affected region (Fig. 3F).

Beginning on Day 4 post-exposure, and coinciding with the onset of grossly visible melanized lesions within the exocuticle (Fig. 4A,B), TSV was detected

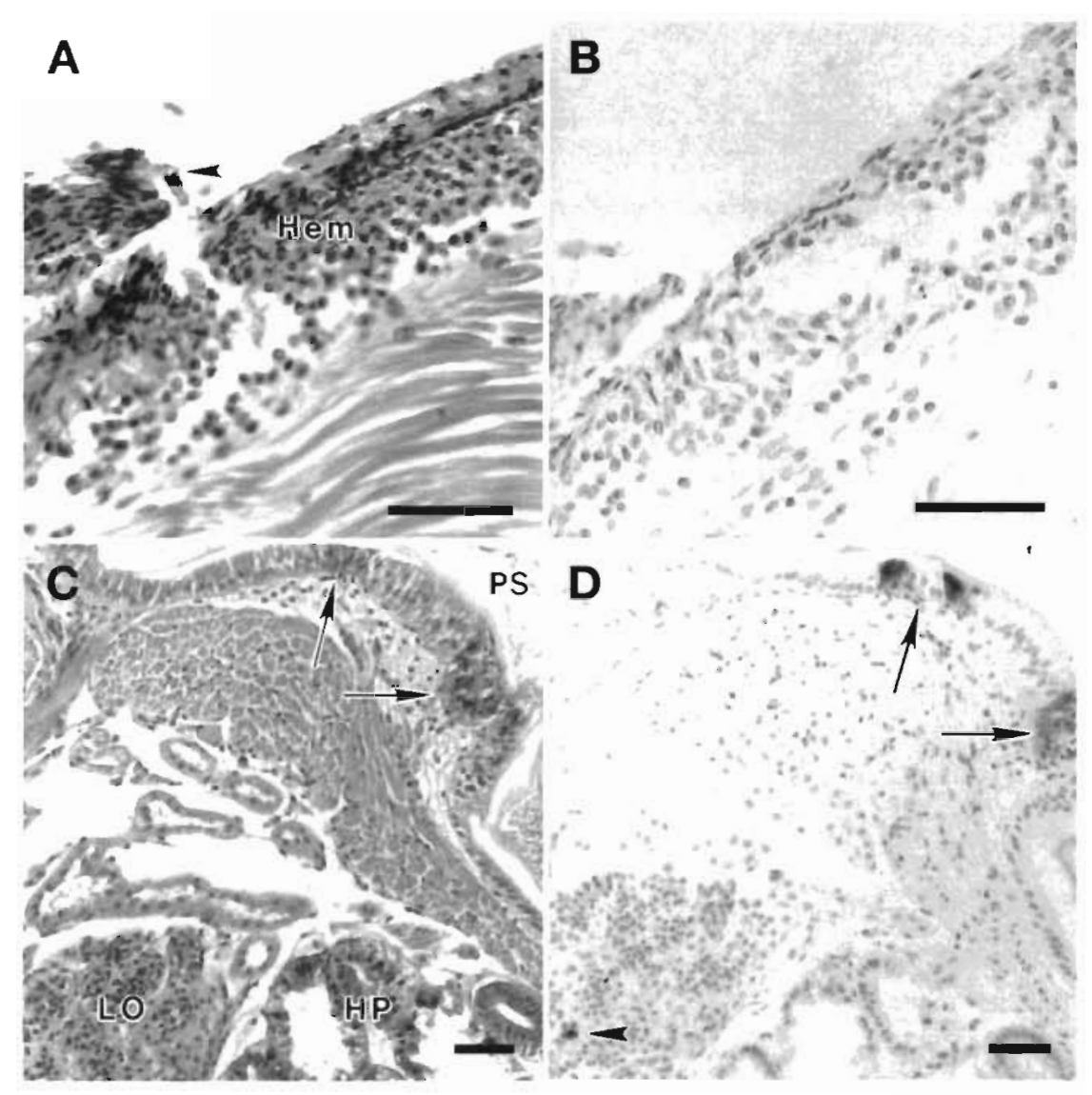

Fig. 4. Penaeus vannamei. Photomicrographs of parallel H\&E-stained (left column) and gene-probed (right column) histological sections illustrating the characteristics of the transitional period (transition phase) of TSV lesion development that occurs between the end of the acute phase and onset of the chronic phase infection periods in juveniles. (A) H\&E stained and (B) gene-probed parallel histological sections of melanized, resolving acute phase TSV lesions within the dorsal cuticle of the tail. A melanized cap composed of flattened hemocytes is partially visible (arrowhead) along with abundant hemocytes (Hem) that have infiltrated a site that previously contained acutely infected cuticular epithelial cells. Rarely were these lesions observed to contain detectable TSV by gene probe analysis, as indicated by the absence of a black precipitate within the inflamed area. (C) H\&E-stained and (D) gene-probed parallel midsagittal histological sections illustrating portions of the lymphoid organ (LO), hepatopancreas (HP) and posterior stomach (PS). Acute phase TSV lesions of the posterior stomach cuticular epithelium are apparent both histologically and by gene probe analysis (arrows). Histologically, the LO appears normal, however, a focal TSV positive signal is evident (arrowhead) in the gene-probed section. Not visible in this photo is the diffuse probe signal present within some of the LO tubules. Gene-probed sections were counter-stained with Bismarck brown. Scale bars $=50 \mu \mathrm{m}$ 
by in situ hybridization analysis within both cuticular epithelial cells and the lymphoid organ (LO) in 1 of the treatment shrimp (Table 2). Observation of TSVpositive probe signals in both foci of acutely infected cuticular epithelial cells and the LO continued up
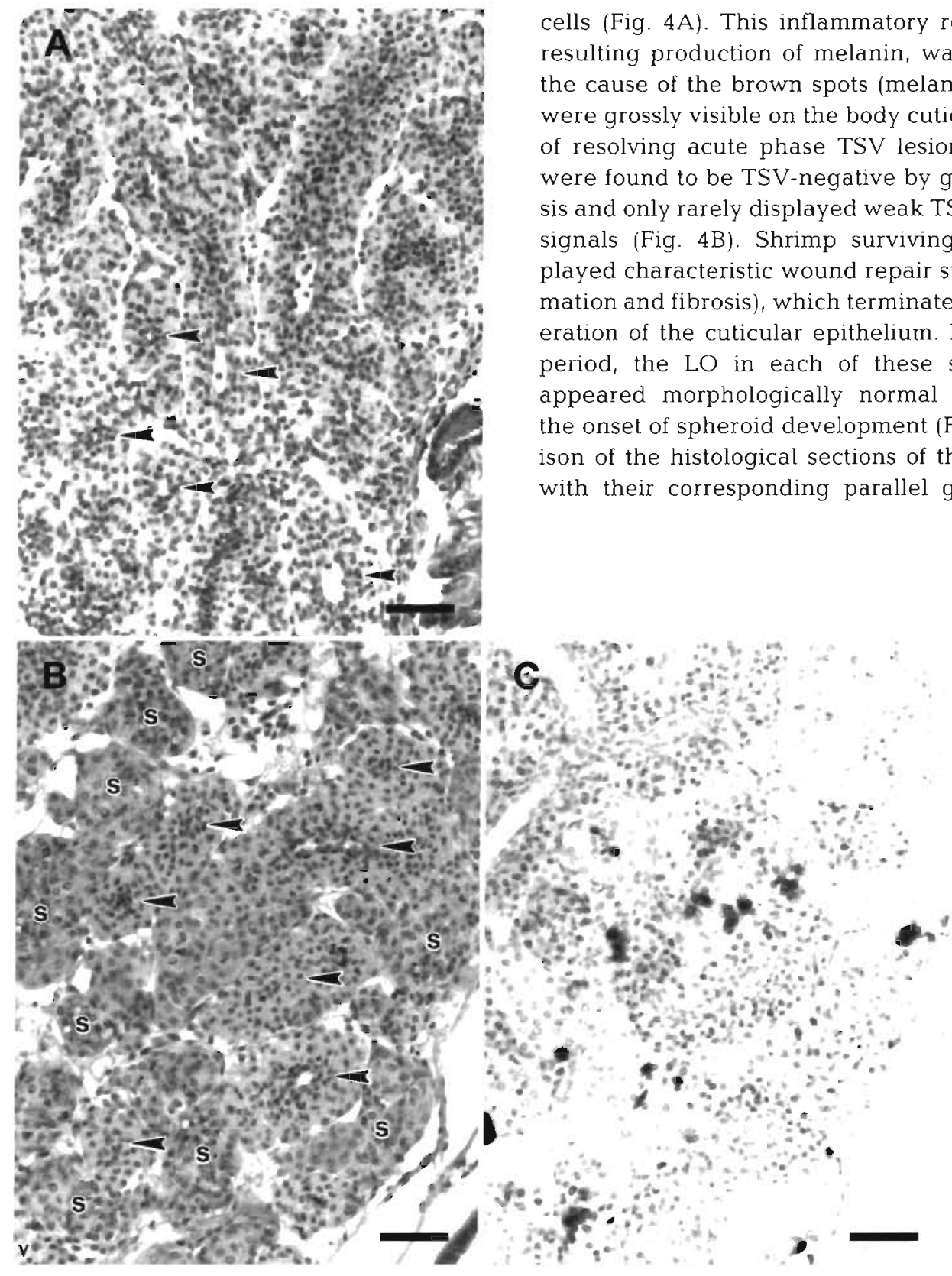

Fig. 5. Penaeus vannamei. Photomicrographs comparing histological sections of a normal lymphoid organ (LO) with one that is chronically infected with TSV. (A) H\&E-stained midsagittal histological section of a normal, uninfected LO. Transversely sectioned LO tubules (arrowheads) consist of an eosinophilic wall of sheath cells and a centralized lumen. (B) H\&E-stained and (C) gene-probed parallel midsagittal histological sections of a chronically infected LO displaying numerous spheroids (s) interspersed among normal appearing LO tubules (arrowheads). Spheroids appear as well-delineated variably sized and shaped masses of presumed sheath cells that are lightly basophilic and lack a centralized lumen. In situ hybridization analysis demonstrates the focal presence of TSV within the LO spheroids, as indicated by the black precipitate. Gene-probed sections were counter-stained with Bismarck brown. Scale bars $=50 \mu \mathrm{m}$

through Day 8 and was detected in a total of 7 shrimp (Table 2). Histological analysis of these 7 samples demonstrated a decrease in the number and severity of acutely infected regions over this $5 \mathrm{~d}$ period and a concurrent increase in the number of sites containing infiltrating hemocytes and melanin deposition within foci that once contained TSV-infected cuticular epithelial cells (Fig. 4A). This inflammatory response, and the resulting production of melanin, was observed to be the cause of the brown spots (melanized lesions) that were grossly visible on the body cuticle and indicative of resolving acute phase TSV lesions. These lesions were found to be TSV-negative by gene probe analysis and only rarely displayed weak TSV-positive probe signals (Fig. 4B). Shrimp surviving this phase displayed characteristic wound repair stages (i.e. inflammation and fibrosis), which terminated with the regeneration of the cuticular epithelium. During this same period, the LO in each of these specimens either appeared morphologically normal or demonstrated the onset of spheroid development (Fig. 4C). Comparison of the histological sections of these 7 specimens with their corresponding parallel gene-probed sec- 
tions showed the presence of either a diffuse TSV-positive gene probe signal within the peripheral sheath cells of morphologically normal appearing LO tubules (not shown) and/or intense focal TSV signals within LO spheroids, when present (Fig. 4D).

By Day 8, mortalities among the positive control group had ceased and the majority of the surviving shrimp had molted, shedding the cuticular melanized lesions along with the old exocuticle. These survivors appeared healthy, i.e. feeding, swimming and, in general, behaving like normal $P e$ naeus vannamei. The only histological abnormality observed within the treatment shrimp during this time period was the presence of numerous spheroids within the LO, which were found to contain low grade to moderate numbers of TSV-infected foci by in situ hybridization analysis (Fig. 5). This lesion type was observed in a total of 15 treatment shrimp that were collected between Days 6 and 10 (Table 2).

The cumulative findings suggested that there were 3 overlapping, but distinct, phases of the TSV disease cycle: a peracute to acute phase, a brief transitional period (named the transition phase) that occurs between the acute and chronic phases of the disease, and finally, a true chronic phase infection. The acute phase disease is characterized by the presence of TSV within 1 or more of the 5 regions containing cuticular epithelium. Shrimp in the transition phase demonstrated TSV-positive probe signals in both the cuticular epithelium and LO and those specimens displaying TSV-positive probe signals solely within the LO were considered to be chronically infected. Utilizing these criteria, each of the 92 geneprobe-positive treatment samples was classified under 1 of the 3 disease phase categories (Table 2). The prevalence of TSV-positive shrimp per disease phase was then determined and plotted over time (Fig. 6).

\section{DISCUSSION}

Early histological analyses of TSV lesion pathogenesis in Penaeus vannamei resulted in the description of a 2-phase infection cycle consisting of a peracute to acute phase and a separate chronic or recovery phase (Lightner et al. 1994, 1995, Brock et al. 1995, Hasson et al. 1995, Lightner 1996a). These findings were based on the observation of lesions in both naturally infected shrimp and experimentally infected moribund shrimp that were collected during short-term infectivity studies. The present investigation represents the first attempt to analyze the TSV disease cycle, utilizing the techniques of both routine H\&E histology and in situ hybridization analysis, in experimentally infected, time-course sampled SPF $P$. vannamei. In this study, the TSV disease cycle in $P$. vannamei was found to consist of 3 distinct, overlapping phases: a peracute to acute phase (hereafter referred to as the acute phase), a short-term transition phase and a definitive chronic phase (Fig. 7). Although the fate of chronically infected $P$. vannamei was not determined in the present investigation, a separate 12 mo time-course study was conducted to address this question (Hasson 1998, Hasson et al. in press). Histological and in situ hybridization results of experimentally infected $P$. vannamei TSV survivors demonstrated that TSV-positive lymphoid organ spheroids persist for at least 8 mo in this penaeid species. This finding confirms the existence of a longterm, TSV-induced chronic phase infection and further supports the 3 phase infection cycle as presented.

\section{Acute phase TSV infection}

Target tissues

The TSV disease cycle begins with an acute phase infection, which is characterized histologically by patho- 


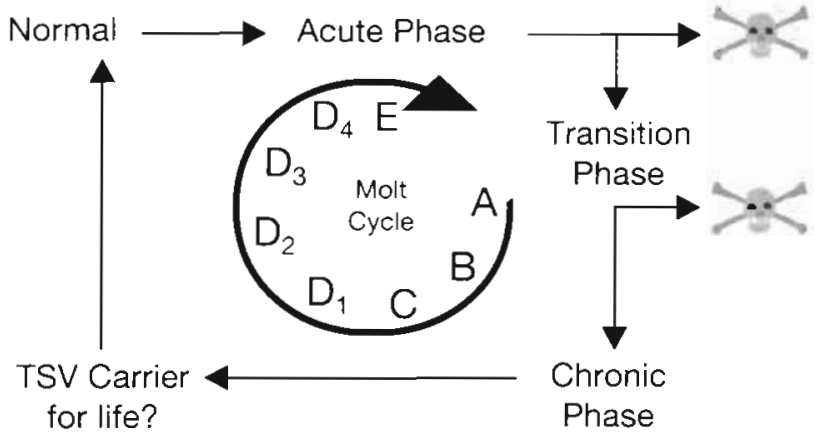

Fig. 7. Penaeus vannamei. TSV disease cycle in juveniles. The acute phase infection occurs during the first $7 \mathrm{~d}$ following per os exposure and is characterized by severe mortalities, cuticular epithelial necrosis and infected shrimp typically dying just prior to (pre-ecdysis, stage D4) or following molt (ecdysis, stage E). The transition phase infection follows, occurring between Days 4 and 8 in a per os-infected population. Characteristics of this TSV disease phase include moderate mortality rates, probe-positive TSV signals within the lymphoid organ (LO), the onset of LO spheroid development, and the presence of both active and resolving acute phase cuticular epithelial lesions, the latter of which are present as grossly visible melanized lesions of the exocuticle. The molt cycle appears to be temporarily inhibited during this stage as the acute phase lesions resolve. Following molt (post-ecdysis, stage A), the shrimp enter the chronic phase of the disease, which is characterized by a cessation of mortalities, resumption of normal behavior, marked LO spheroid development and the presence of detectable TSV within LO spheroids by gene probe analysis. It is unclear from the present study if chronic phase TSV infections persist for the remainder of the host's life or are eventually resolved with a return to normalcy. Molt cycle stages after Roer \& Dillaman (1993)

diagnostic, severe multifocal to diffuse necrosis and nuclear pyknosis/karyorrhexis of the cuticular epithelial cells and subcutis located in the 5 targeted regions (Fig. 2). These lesions are detectable by in situ hybridization as early $24 \mathrm{~h}$ post-per os exposure and were observed for up to $7 \mathrm{~d}$. Notably absent during this phase of the disease was a detectable inflammatory response by the shrimp host. Severe lesions were occasionally observed to extend into the underlying subcuticular connective tissue or striated muscle as previously reported (Lightner et al. 1994, 1995, Brock et al. 1995). In brief, the histological characteristics of the acute phase TSV lesion type observed in this study corroborate those previously described by Jimenez (1992), Lightner et al. (1994, 1995), Brock et al. (1995, 1997) and Hasson et al. $(1995,1997,1999)$.

Although the highest and lowest prevalence of acute phase lesions was observed within the cuticular epithelium of the foregut and hindgut, respectively, it appears that the virus is not highly site selective, infecting cuticular epithelial cells at random without showing an obvious preference for one region of this cell type over another. Plausible reasons for the high infection prevalence observed in the foregut cuticular epithelium include the possibility that the virus enters its host through the gut wall and that this is the first region of the shrimp that is exposed to ingested TSVinfected tissue. One can speculate that the dispersion of the virus to uninfected regions of cuticular epithelial cells then occurs at random and is facilitated by hemolymph circulation. However, neither the mechanism involved nor the site of TSV entry into the shrimp was revealed in the present study.

Histological and/or gene probe analyses of SPF Penaeus vannamei that were injected with purified TSV or TSV-infected tissue homogenates during previous infectivity studies have demonstrated that acute phase TSV lesions are, occasionally, observed histologically or detected by gene probe analysis within hematopoietic nodules, the male reproductive tract, and the antennal gland (Hasson et al. 1995, Hasson 1998). However, TSV lesions within these organs were only observed within test shrimp with severe injectioninduced TSV infections, but were not observed in the per os-exposed test shrimp of the present study nor have they been reported in naturally infected $P$. vannamei. As a result, TSV infection of these organs may or may not prove to be of significance.

Acute phase TSV infections result in 3 stages of cell necrosis that are observable by light microscopy (Fig. 3). During the first 2 stages, infected cuticular epithelial cells display nuclear pyknosis and eosinophilia of the cytoplasm. The second stage of necrosis is distinguished from the first by the presence of irregularly shaped to circularized cells that have detached from the surrounding cell matrix. In both Stages 1 and 2, the infected cells remain intact and produce a strong TSV probe signal by in situ hybridization analysis. The third and final stage of acute phase TSV-induced necrosis results in nuclear fragmentation and cell lysis. Viewed as the pathodiagnostic histological lesion type of an acute phase TSV infection, this ultimate stage of TSV-induced necrosis is the least desirable for gene probe analysis as the virus appears to be rapidly dispersed following cell lysis. This, in part, helps to explain the inability and difficulty of early investigations into the etiology of TS to identify the presence of TSV virions within acute phase lesions by transmission electron microscopy (Lightner et al. 1994, 1995). In these early investigations, it was standard practice to prepare thin sections of tissues containing pathodiagnostic acute phase TSV lesions, which we now know contain little or no detectable viral particles.

\section{Clinical signs}

The clinical signs of acute phase TSV infections have been well documented in Penaeus vannamei with 
either naturally occurring or experimentally induced infections (Lightner et al. 1994, 1995, Brock et al. 1995, Hasson et al. 1995, 1997, 1999, Lightner 1996a). The clinical findings of the present study coincide with those previously observed in $P$. vannamei following per os or injection-mediated TSV exposure. Typically, acutely infected $P$. vannamei die during or immediately post-molt, suggesting that the molt cycle is intimately linked to an acute phase TSV infection. Previous studies have shown that acutely infected moribund shrimp are, generally, in the late D4 to E stage at the time of death (Lightner 1996a). The soft texture of the cuticle at time of death is likely the result of partial resorption of calcium carbonate in preparation for the impending molt (Roer \& Dillaman 1993). The cuticle synthesizing activity of the cuticular epithelial cells during this premolt stage may be a prerequisite for successful viral replication and, perhaps, explains the correlation between soft cuticles and severe acute phase TSV infection in $P$. vannamei. Although the relationship between molt stage and TSV-induced death was not analyzed, it appears that death or recovery from the disease is dependent upon the molt stage, molt frequency, and the inherent viral resistance of the infected shrimp. It is during this stage of the TSV disease cycle that susceptible late postlarval and juvenile $P$. vannamei populations suffer cumulative mortalities ranging from 75 to $95 \%$. In the case of the positive control treatment shrimp, a cumulative percent mortality of $85 \%$ resulted by the end of the $10 \mathrm{~d}$ experiment with mortalities having peaked on Day 4 post-per os exposure. Comparison of the positive control shrimp mortality curve (Fig. 1) with that of the graph illustrating the daily percentage of acutely infected treatment shrimp (Fig. 6) shows that peak acute phase mortalities coincide with peak acute phase lesion prevalence (Day 4 post-exposure). These results suggest that the mortalities are directly attributable to the acute phase TSV lesions.

Depending on the infectious dose of TSV consumed by, or injected into, Penaeus vannamei test shrimp, the time frame post-exposure when peak mortalities are observed will vary from one bioassay to another. In this per os study, peak mortality occurred on Day 4, whereas previous TSV per os infectivity studies have reported peak mortalities on Days 5 or 6 (Brock et al. 1995, Lotz 1997, Overstreet et al. 1997). With injection-mediated TSV studies, the time frame to peak mortality is even shorter, occurring within 2 to $3 \mathrm{~d}$ post-injection (Brock et al. 1995, Hasson 1998). The amount of infected tissue fed in the present study ( $34 \%$ of the biomass $d^{-1}$ for $3 \mathrm{~d}$ ) greatly exceeded the standard amount (10 to $20 \%$ of the biomass) typically fed; this was done with the objective of infecting the entire population of each aquarium rapidly and in unison. This appears to account for the shorter time interval to peak mortality. As a result, the time frames for each of the infection cycles described herein should be viewed as approximations when considering the cycle of a naturally occurring TSV epizootic.

\section{Transition phase TSV infection}

Penaeus vannamei surviving the acute phase disease enter into a transitional infection period, which shares histological attributes of both the acute and chronic phases of the disease cycle and, in effect, links them together (Fig. 4). This stage of infection was previously referred to as the chronic or recovery phase by Lightner et al. (1995) and Hasson et al. (1995). However, since the present findings suggest that TSV-infected shrimp do not fully recover from the disease during this period and, furthermore, indicate that a true active chronic infection ensues, it is proposed that the name 'transition phase' would be more descriptive and accurate. The transition phase begins approximately $4 \mathrm{~d}$ post-per os exposure, overlaps with both the acute and chronic phases, lasts for $-5 \mathrm{~d}$, and has 3 defining histological characteristics. Grossly, the shrimp display numerous multifocal, variably sized and shaped melanized cuticular lesions throughout both the cephalothorax and abdominal regions. Histologically, these lesions consist of melanized hemocytic infiltrates, which represent resolving acute phase lesions (Fig. 4). Occurring concurrently is the continued presence of a few active focal acute phase lesions within the cuticular epithelium and the onset of lymphoid organ spheroid formation. By in situ hybridization analysis, normal appearing LO tubules display a diffuse TSV-positive gene probe signal, ranging in intensity from weak to strong, throughout the periphery of the sheath cell wall. This finding is suggestive of active uptake or sequestering of the virus by the LO sheath cells. When present, LO spheroids may display strong focal TSV-positive probe signals (Fig. 4). The virus is also detectable in the few remaining acute phase lesions that remain, but absent in foci displaying advanced melanization. As indicated in Fig. 2, shrimp mortalities still occur during this phase of the infection cycle, but are on the decline. Another distinguishing feature of the transition phase is that the molt cycle appears to be temporarily suppressed or inactivated during this period as new cuticular epithelial cells regenerate. Surviving shrimp then complete the molt cycle, shedding the melanized lesions along with the old cuticle and enter into the chronic phase of the disease cycle (Fig. 5). 


\section{Chronic phase TSV infection}

Chronic phase TSV infection overlaps with both the acute and transition phases and begins $\sim 6 \mathrm{~d}$ following per os exposure (Fig. 7). Chronically infected Penaeus vannamei resume normal behavioral patterns and display no observable clinical signs of TSV infection, and there is a cessation of TSV-induced mortalities. The defining histological characteristics of this phase are the complete absence of acute phase histological lesions of the cuticular epithelium and marked LO hypertrophy directly resulting from the rapid development of numerous LO spheroids, some of which are TSV positive by in situ hybridization analysis.

LO spheroid development has been associated with at least 4 other penaeid shrimp viral infections: lymphoid organ vacuolization virus (LOVV, Bonami et al. 1992), lymphoidal parvo-like virus (LPV. Owens et al. 1991), rhabdovirus of penaeid shrimp (RPS, Nadala et al. 1992) and lymphoid organ virus (LOV, Spann et al. 1995). As originally hypothesized by Hasson et al. (1995), the development of LO spheroids was confirmed to be TSV-induced in the present study and not due to LOVV infection as originally reported (Jimenez 1992). However, it remains unclear from the present study if LO spheroids represent a host-mediated immune response to infection, simply a generalized response by this tissue type to infection-mediated injury, or a combination of the two. As LO spheroid development occurs with a variety of infections, a definitive diagnosis of their etiology cannot be made by routine histology, but requires the application of virus-specific gene probes. In the case of TSV, we have demonstrated herein that diagnosis of asymptomatic, chronically TSV-infected shrimp is feasible utilizing in situ hybridization analysis.

Acknowledgements. Support for this investigation was through grants from the Gulf Coast Research Laboratory Consortium Marine Shrimp Farming Program, CSREES, U.S. Dept of Agriculture (grant no. 95-38808-1424), the National Sea Grant Program, U.S. Dept of Commerce, NOAA (grant no. NA56FD0621), and by the National Fisheries Institute.

\section{LITERATURE CITED}

Bell TA, Lightner DV (1987) IHHN disease of Penaeus stylirostris: effects of shrimp size on disease expression. J Fish Dis 10:165-170

Bell TA, Lightner DV (1988) A handbook of normal penaeid shrimp histology. World Aquaculture Society, Baton Rouge

Bonami JR, Lightner DV, Redman RM, Poulos BI (1992) Partial characterization of a togavirus (LOVV) associated with histopathological changes of the lymphoid organ of penaeid shrimps. Dis Aquat Org 14:145-152

Bonami JR, Hasson KW, Mari J, Poulos BT, Lightner DV (1997) Taura syndrome of marine penaeid shrimp: characterization of the viral agent. J Gen Virol 78:313-319
Brock JA (1997) Special topic review: Taura syndrome, a disease important to shrimp farms in the Americas. World $\mathbf{J}$ Microbiol Biotechnol 13:415-418

Brock JA, Gose RB, Lightner DV, Hasson KW (1995) An overview on Taura syndrome, an important disease of

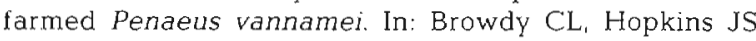
(eds) Swimming through troubled water. Proceedings of the special session on shrimp farming. Aquaculture ' 95 . World Aquaculture Society, Baton Rouge, p 84-94

Brock JA, Gose RB, Lightner DV, Hasson KW (1997) Recent developments and an overview of Taura syndrome of farmed shrimp in the Americas. In: Flegel TW, MacRae IH (eds) Diseases in Asian aquaculture III. Fish health section. Asian Fisheries Society, Manila, p 275-283

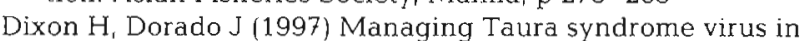
Belize: a case study. Aquacult Mag 23(3):30-42

Hasson KW (1998) Taura syndrome in marine penaeid shrimp: discovery of the viral agent and disease characterization studies. PhD dissertation, University of Arizona, Tucson

Hasson KW, Lightner DV, Poulos BT, Redman RM, White BL, Brock JA, Bonami JR (1995) Taura syndrome in Penaeus vannamei: demonstration of a viral etiology. Dis Aquat Org 23:115-126

Hasson KW, Hasson J, Aubert H, Redman RM, Lightner DV (1997) A new RNA-friendly fixative for the preservation of penaeid shrimp samples for virological detection using cDNA genomic probes. J Virol Methods (66):227-236

Hasson KW, Lightner DV, Mari J, Bonami JR, Poulos BT, Mohney LL, Redman RM, Brock JA (1999) The geographic distribution of Taura syndrome virus (TSV) in the Americas: determination by histopathology and in situ hybridization using TSV-specific cDNA probes. Aquaculture $171: 13-26$

Hasson KW, Lightner DV, Mohney LL, Redman RM, White BM (1999) Taura Syndrome Virus (TSV) chronicity and spheroid development in Penaeus vannamei: a possible cell-mediated immune response to a persistent viral infection. Dis Aquat Org (in press)

Jimenez R (1992) Sindrome de Taura (Resumen). Acuacultura del Ecuador 1:1-16

Lightner DV (1995) Taura syndrome: an economically important viral disease impacting the shrimp farming industries of the Americas including the United States. In: Campbell $P$ and Associates (eds) Proceedings of the 99th Annual Meeting USAHA, Reno, NV. P. Campbell and Associates, Richmond, p 36-52

Lightner DV (1996a) A handbook of shrimp pathology and diagnostic procedures for diseases of cultured penaeid shrimp. World Aquaculture Society, Baton Rouge

Lightner DV (1996b) Epizootiology, distribution, and the impact on international trade of two penaeid shrimp viruses in the Americas. Rev Sci Tech OIE (Off Int Epizoot) 15(2):579-601

Lightner DV, Redman RM (1985) A parvo-like disease of penaeid shrimp. J Invertebr Pathol 45:47-53

Lightner DV, Jones LS, Ware GW (1994) Proceedings of the Taura syndrome workshop; executive summary, submitted reports, and transcribed notes. Jan 21-22, 1994. University of Arizona, Tucson

Lightner DV, Redman RM, Hasson KW, Pantoja CR (1995) Taura syndrome in Penaeus vannamei: histopathology and ultrastructure. Dis Aquat Org 21:53-59

Lightner DV, Redman RM, Poulos BT, Nunan LM, Mari JL, Hasson KW, Bonami JR (1997) Taura syndrome: etiology, pathology, hosts and geographic distribution, and detection methods. In: New approaches to viral diseases of 
aquatic animals. Proceedings of the NRIA international workshop, Jan 21-24, Kyoto, p 190-205

Lotz (1997) Effect of the host size on virulence of Taura Virus to the marine shrimp Penaeus vannamei (Crustacea: Penaeidae). Dis Aquat Org 30:45-51

Mari J, Bonami JR, Lightner DV (1998) Taura syndrome of penaeid shrimp: cloning of viral genome fragments and development of specific gene probes. Dis Aquat Org 33 $11-17$

Nadala ECB, Lu Y, Loh PC, Brock JA (1992) Infection of Penaeus stylirostris (Boone) with a rhabdovirus isolated from Penaeus sp. Gyobyo Kenekyu 27:143-147

Overstreet RM, Lightner DV, Hasson KW, Mcrlwain S, Lotz J (1997) Susceptibility to TSV of some penaeid shrimp native to the Gulf of Mexico and southeast Atlantic Ocean J Invertebr Pathol 69:165-176

Owens L, De Beer S, Smith J (1991) Lymphoidal parvoviruslike particles in Australian penaeid prawns. Dis Aquat Org 11:129-134

Perez Farfante I, Kensley BF (1997) Penaeoid and sergestoid shrimps and prawns of the world: keys and diagnoses for

Editorial responsibility: Timothy Flegel.

Bangkok, Thailand the families and genera. Mem Museum Natl Hist Nat 175:1-233

Pruder GD, Brown CL, Sweeney JN, Carr WH (1995) High health shrimp systems: seed supply, theory and practice In: Browdy CL, Hopkins JS (eds) Swimming through troubled water Proceedings of the special session on shrimp farming. World Aquaculture Society, Baton Rouge, p $40-52$

Roer RD, Dillaman RM (1993) Molt related change in integumental structure and function. In: Horst MN, Freeman JA (eds) The crustacean integument: morphology and biochemistry. CRC Press, Boca Raton, p 1-37

Spann KM, Vickers JE, Lester RJ (1995) Lymphoid organ virus of Penaeus monodon from Australia. Dis Aquat Org 23:127-134

Wyban JA, Swingle JS, Sweeney JN, Pruder GD (1992) Development and commercial performance of high health shrimp using specific pathogen free (SPF) broodstock Penaeus vannamei. In: Wyban JA (ed) Proceedings of the special session on shrimp farming. World Aquaculture Society, Baton Rouge, p 254-259

Submitted: September 18, 1998; Accepted:December 11, 1998 Proofs received from author(s): April 9, 1999 